\title{
Presence of Serum Antinuclear Antibodies Does Not Impact Outcomes in HBV-Related Acute-on-Chronic Liver Failure
}

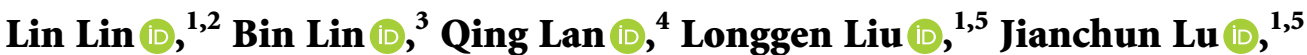 \\ Xiujun Zhang $\mathbb{D}^{1,5}$ Shuqin Zheng $\mathbb{D}^{1,5}$ and Yuan Xue $\mathbb{D}^{1,5}$ \\ ${ }^{1}$ Institute of Hepatology, The Third People's Hospital of Changzhou, Changzhou, China \\ ${ }^{2}$ Department of Pharmacy, The Third People's Hospital of Changzhou, Changzhou, China \\ ${ }^{3}$ Department of Infectious Diseases, The First People's Hospital of Jintan, Changzhou, China \\ ${ }^{4}$ Department of Infectious Diseases, The First Affiliated Hospital of Kunming Medical University, Kunming, Yunnan, China \\ ${ }^{5}$ Department of Liver Diseases, The Third People's Hospital of Changzhou, Changzhou, China
}

Correspondence should be addressed to Yuan Xue; xueyuan80908@163.com

Received 20 November 2021; Revised 16 January 2022; Accepted 26 January 2022; Published 18 February 2022

Academic Editor: Alessandro Granito

Copyright (c 2022 Lin Lin et al. This is an open access article distributed under the Creative Commons Attribution License, which permits unrestricted use, distribution, and reproduction in any medium, provided the original work is properly cited.

\begin{abstract}
Background. The aim of this study was to provide new insights into the prevalence of positive antinuclear antibody (ANA) in patients with HBV-related acute-on-chronic liver failure (ACLF) and its impact on clinical outcomes. Methods. A total of 116 patients with HBV-related ACLF treated at three clinical centers were retrospectively recruited. Serum concentrations of ANA were detected using the enzyme-linked immunosorbent assay kit. Multiple nuclear dots, rim-like, and centromere patterns of ANA were detected using indirect immunofluorescence assay on HEp-2 cells. Results. Among the 116 patients with HBV-related ACLF, 17 (14.66\%) were ANA positive. Most patients in both ANA positive and negative groups were males (88.2\% and 83.8\%). Patients with negative ANA had a higher international normalized ratio, model for end-stage liver disease (MELD), and MELDsodium scores than those with positive ANA (all $P<0.05)$. Multiple nuclear dot pattern was detected in half of the patients $(8 / 17$, 47.06\%), rim-like/membranous pattern was found in six patients, and centromere pattern was detected in the last three patients. For patients with ANA (+), IgM was lower, and it was positively correlated with IgG. For patients with ANA (-), C3 was positively correlated with C4, and both C3 and C4 were negatively correlated with INR and MELD (all $P<0.05)$. In addition, TBIL, INR, WBC, and PLT, but not ANA, resulted as independent risk factors associated with 90-day mortality. Conclusion. Positive ANA is frequent in HBV-related ACLF, and it does not seem to be associated with poor outcomes, but the pathogenesis of ACLF may be different between ANA (+) and ANA (-) groups.
\end{abstract}

\section{Introduction}

Acute-on-chronic liver failure (ACLF) is a life-threatening syndrome resulting from an acute hepatic insult in patients with chronic liver diseases. Hepatitis B virus (HBV)-related ACLF (HBV-ACLF) is characterized by acute hepatic deterioration and high short-term mortality in patients with chronic hepatitis B (CHB) or liver cirrhosis (LC) [1]. Although definitions of ACLF differ among European, North America, and Asian Pacific countries, it is important to emphasize that systematic inflammation has a critical role in the pathophysiology and outcome of ACLF [2]. Moreover, according to the definition by the Asian Pacific Association for the Study of the Liver (APASL), intrahepatic precipitating events such as HBV reactivations are of the utmost importance, while extrahepatic disorders, including bacterial infections, are considered as a complication, but not a trigger of ACLF.

The issue of an antinuclear antibody (ANA) has received considerable critical attention in chronic liver disease $[3,4]$. It has been reported that $21 \%$ of patients with nonalcoholic fatty liver disease (NAFLD) had positive ANA [5, 6]. Another study revealed that patients with positive ANA did not exhibit histological features of autoimmune hepatitis (AIH) or developed 
AIH during follow-up. Moreover, $21.8 \%$ of patients with chronic hepatitis $\mathrm{C}$ had positive ANA and lower sustained virological response to interferon-based therapy, while the incidence of cirrhosis, hepatocellular carcinoma, and mortality showed no significant difference from those with negative ANA [7]. It seems that long-term outcomes of NASH and chronic hepatitis $\mathrm{C}$ are not affected by ANA $[7,8]$. To the best of our knowledge, the prevalence of ANA and its impact on clinical outcomes remain largely unknown in patients with HBV-related ACLF. Consequently, it could be useful to study whether the ANA positivity serves as an additional intrahepatic precipitating event.

This study provided new insights into the prevalence of positive ANA in patients with HBV-related ACLF and its impact on clinical outcomes.

\section{Materials and Methods}

2.1. Patients and the Primary Endpoint. A total of 116 patients with HBV-ACLF treated in three clinical centers were prospectively recruited between January 2015 and October 2021. ACLF was diagnosed according to the criteria from APASL [2]. Patients were excluded if they had a drug-induced liver injury, liver cancer, or coinfection with other hepatitis virus or human immunodeficiency virus. Patients with suspicious autoimmune hepatitis (AIH) or primary biliary cirrhosis (PBC) were excluded according to the simplified criteria for the diagnosis of $\mathrm{AIH}$ and the guideline from the British Society of Gastroenterology and UK-PBC [9-11]. Data including age, sex, survival time, alanine transaminase, aspartate transaminase, total bilirubin (TBil), international normalized ratio (INR), creatinine, HBV serologic markers, platelets count, white blood cells count, and complications, were collected.

The study was approved by the Ethics Committee of the Third People's Hospital of Changzhou according to the Declaration of Helsinki 1975, and the informed consent was obtained from all participants.

2.2. Enzyme-Linked Immunosorbent Assay. Serum concentrations of ANA were detected using the enzyme-linked immunosorbent assay kit (YHLO Biotech Co., Ltd., Shenzhen, Guangdong, China). Serum was initially diluted at 1:80 in PBS and tested; then, the positive serum was serially diluted (from $1: 160$ to $1: 1280)$. Serum with a titer of $>1: 80$ was reported as positive and tested for binding site. Multiple nuclear dots, rimlike, and centromere (MRC) patterns of ANA were detected using indirect immunofluorescence assay on HEp-2 cells according to the manufacturer's instructions (Medical and Biological Laboratories, Aichi-Ken, Japan). Serum concentrations of immunoglobulin (Ig) $\mathrm{G}$ and $\mathrm{M}$ and complement C3 and $\mathrm{C} 4$ were measured using the enzyme-linked immunosorbent assay kit (Medical system Biotechnology Co., Ltd., Ningbo, Zhejiang, China).

2.3. Score Systems. The model for end-stage liver disease (MELD) [12] and MELD-Na [13] scores were calculated as previously described.
2.4. Statistical Analysis. All data were analyzed using SPSS version 25.0 (Chicago, IL, USA). Continuous variables were expressed as median with range and compared using the Mann-Whitney $U$ test. Categorical values were presented as frequencies and compared using the chi-square test. Correlation analysis was evaluated using the Spearman correlation test. Survival analysis was performed using the Kaplan-Meier curve. Independent risk factors for 90-day mortality were identified using logistic regression analysis. A two-sided $P<0.05$ was considered statistically significant.

\section{Results}

3.1. Characteristics of Patients. The characteristics of the 116 patients with HBV-related ACLF are given in Table 1. Among these patients, 17 (14.66\%) were ANA positive (Figure 1). Most patients in both ANA positive and negative groups were males $(88.2 \%$ and $83.8 \%, P>0.99)$. Patients with negative ANA had higher INR, MELD, and MELD-Na scores than those with positive ANA (all $P<0.05$ ). There was no significant difference in the incidence of spontaneous bacterial peritonitis (SBP) and hepatic encephalopathy (HE) between the two groups $(P>0.05)$. None of those patients from both groups were diagnosed with autoimmune hepatitis or connective tissue disease.

Among the 17 patients with positive ANA, 11 (64.71\%) had a titer of $1: 160$, and the other six patients had $1: 320$. Multiple nuclear dot pattern was detected in half of the patients $(8 / 17,47.06 \%)$, rim-like/membranous pattern was found in six patients, and centromere pattern was detected in the last three patients.

3.2. Concentrations of IgG, IgM, and Complement C3 and C4. IgG, IgM, C3, and C4 were detected in 12 ANA (+) patients and 65 ANA (-) patients (Figure 2(a)). IgM in the ANA (+) group was significantly lower compared with that in the ANA $(-)$ group $(P=0.025)$. There was no significant difference regarding IgG, C3, and C4 between ANA (+) and ANA (-) groups (all $P>0.05$ ). As shown in Figure 3, for patients with ANA (-), C3 and C4 were negatively correlated with INR (both $P<0.05$ ), while the correlation was not found in patients with ANA (+). Furthermore, negative correlations between MELD score and C3 and C4 were observed in the ANA (-) group (both $P<0.05$ ), but not in the ANA (+) group. Moreover, IgM was positively correlated with $\operatorname{IgG}(\gamma=0.677, P=0.02)$ in the ANA $(+)$ group, while C3 was positively correlated with $\mathrm{C} 4$ in the ANA (-) group $(\gamma=0.760, P<0.01)$.

3.3. Outcomes of ANA (+) Patients with ACLF. For ANA (+) and ANA (-) patients, the 90 -day mortalities were $29.4 \%$ (5/17) and $37.4 \%$ (37/99), respectively. As shown in Figure 2(b), survival analysis using the Kaplan-Meier method showed no significant differences between ANA (+) and ANA (-) groups during 90-day follow-up $\left(\chi^{2}=0.675, P=0.41\right)$. 
TABLE 1: Characteristics of patients with ACLF.

\begin{tabular}{|c|c|c|c|c|}
\hline Variables & ANA positive $(n=17)$ & ANA negative $(n=99)$ & $Z$ or $\chi^{2}$ & $P$ value \\
\hline Age (years) & $50.0(33.0-59.0)$ & $51.0(41.0-59.0)$ & -0.859 & 0.39 \\
\hline Male, $n(\%)$ & $15(88.2)$ & $83(83.8)$ & 0.214 & $>0.99$ \\
\hline HBeAg, $n(\%)$ & $6(35.3)$ & $32(32.3)$ & 0.058 & 0.81 \\
\hline $\operatorname{ALT}(\mathrm{U} / \mathrm{L})$ & $322.0(156.3-991.3)$ & $272.3(87.0-690.0)$ & -0.972 & 0.33 \\
\hline AST (U/L) & $292.8(120.3-418.7)$ & $178.0(91.4-419.0)$ & -1.296 & 0.20 \\
\hline TBil $(\mu \mathrm{mol} / \mathrm{L})$ & $288.8(201.8-395.8)$ & $302.4(212.5-377.7)$ & -0.121 & 0.90 \\
\hline Creatinine $(\mu \mathrm{mol} / \mathrm{L})$ & $67.1(55.8-80.5)$ & $64.2(54.4-83.9)$ & -0.293 & 0.77 \\
\hline Platelet $(E+09 / \mathrm{L})$ & $102.0(59.5-181.0)$ & $98.0(70.0-134.0)$ & -0.894 & 0.37 \\
\hline WBC $(E+09 / \mathrm{L})$ & $7.1(5.6-9.6)$ & $6.0(4.8-9.3)$ & -1.300 & 0.19 \\
\hline Neutrophils $(E+09 / \mathrm{L})$ & $4.8(3.6-6.5)$ & $4.0(2.9-6.0)$ & -1.261 & 0.21 \\
\hline Serum sodium $(\mathrm{mmol} / \mathrm{L})$ & $139.0(136.7-140.2)$ & $137.6(134.6-139.8)$ & -1.405 & 0.16 \\
\hline INR & $1.8(1.6-2.1)$ & $2.1(1.8-2.7)$ & -2.799 & $<0.01$ \\
\hline MELD score & $24.0(21.5-26.0)$ & $26.0(24.0-29.0)$ & -2.449 & 0.01 \\
\hline MELD-Na score & $25.0(21.5-26.0)$ & $27.0(24.0-31.0)$ & -2.429 & 0.01 \\
\hline Cirrhosis, $n(\%)$ & $10(58.8 \%)$ & $58(58.1 \%)$ & 0.001 & 0.99 \\
\hline $\mathrm{SBP}, n(\%)$ & $5(29.4 \%)$ & $29(29.3 \%)$ & 0.001 & 0.99 \\
\hline $\mathrm{HE}, n(\%)$ & $7(41.2 \%)$ & $44(44.4 \%)$ & 0.063 & 0.80 \\
\hline Death (\%) & $5(29.4 \%)$ & $37(37.4 \%)$ & 0.398 & 0.53 \\
\hline
\end{tabular}

Data were expressed as median (IQR) for continuous variables and $n$ (\%) for categorical values and were compared using the Mann-Whitney $U$ test or chisquare test. ACLF, acute-on-chronic liver failure; ANA, antinuclear antibodies; ALT, alanine aminotransferase; AST, aspartate aminotransferase; TBil, total bilirubin; ALP, alkaline phosphatase; GGT, gamma glutamyl transferase; CHE, cholinesterase; INR, international normalized ratio; HBeAg, hepatitis B e antigen; WBC, white blood cell; MELD, model for end-stage liver disease; SBP, spontaneous bacterial peritonitis; HE, hepatic encephalopathy.

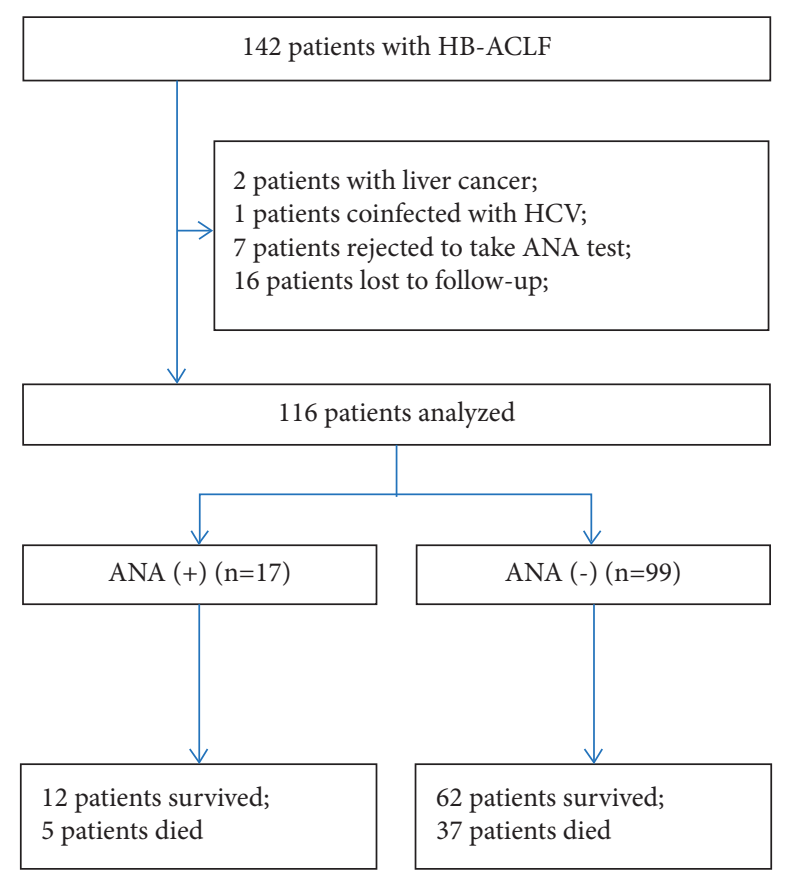

FIGURE 1: Screening of patients with acute-on-chronic liver failure and positive antinuclear antibody.

3.4. Independent Risk Factors for 90-Day Mortality. As given in Table 2, the univariate logistic analysis showed that TBIL, INR, creatinine, serum sodium, WBC, and PLT were associated with 90-day mortality. Multivariable analysis showed that TBIL, INR, WBC, and PLT (all $P<0.05$ ) were independent risk factors for 90-day mortality. Next, an adjusted logistic analysis was performed using the MELD score, instead of TBIL and INR. Data showed that MELD, WBC, and PLT were independent risk factors for 90-day mortality $(P=0.001,0.004$, and 0.023$)$.

\section{Discussion}

The present study investigated the prevalence of positive ANA in patients with HBV-related ACLF and its impact on clinical outcomes. Our data showed that ANA was positive in $14.66 \%$ of patients with HBV-related ACLF. Patients with positive ANA had lower INR, MELD, and MELD-Na scores than those with negative ANA. For patients with ANA (+), IgM was lower, and it was positively correlated with IgG. For patients with ANA (-), C3 was positively correlated with $\mathrm{C} 4$, 


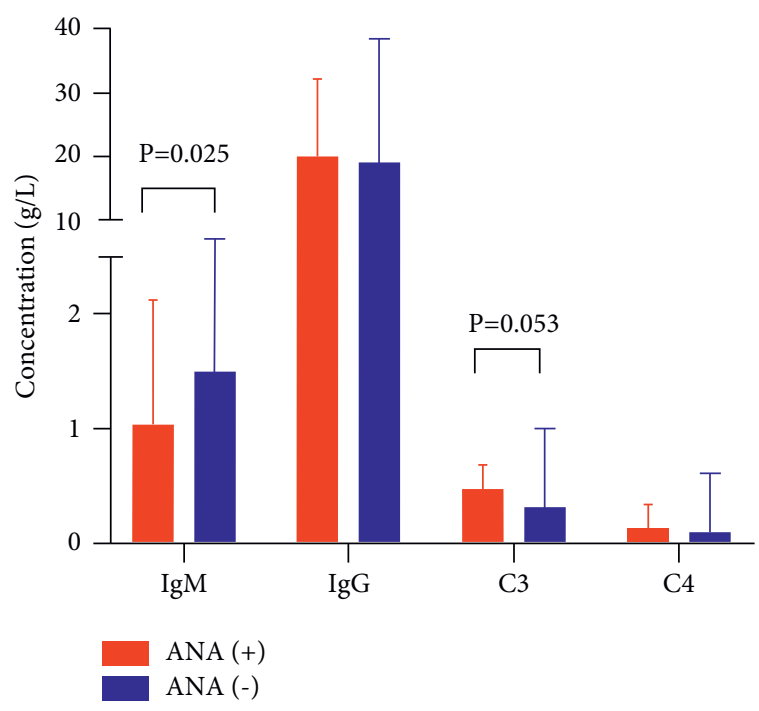

(a)

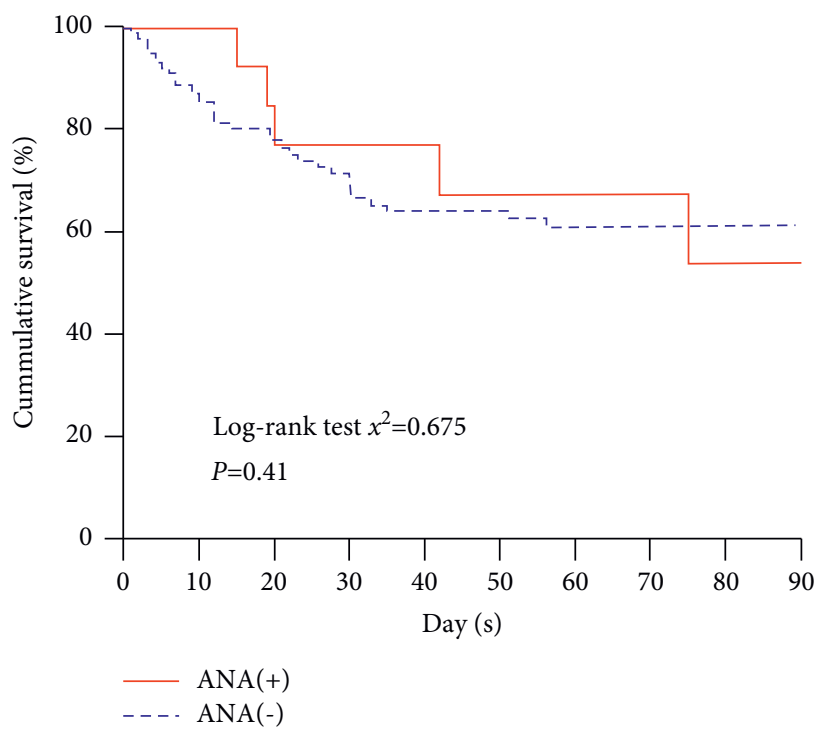

(b)

Figure 2: Comparison of immunoglobulin (Ig), complements, and survival between antinuclear antibody positive and negative patients with HBV-ACLF. (a) Comparison of IgG and IgM and complement C3 and C4. (b) Kaplan-Meier survival analysis between ANA (+) and ANA (-) groups during 90-day follow-up.

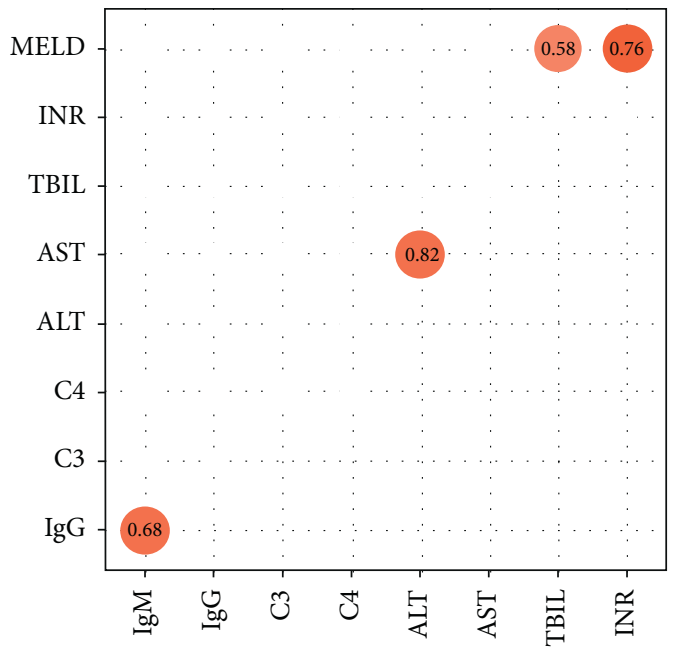

(a)

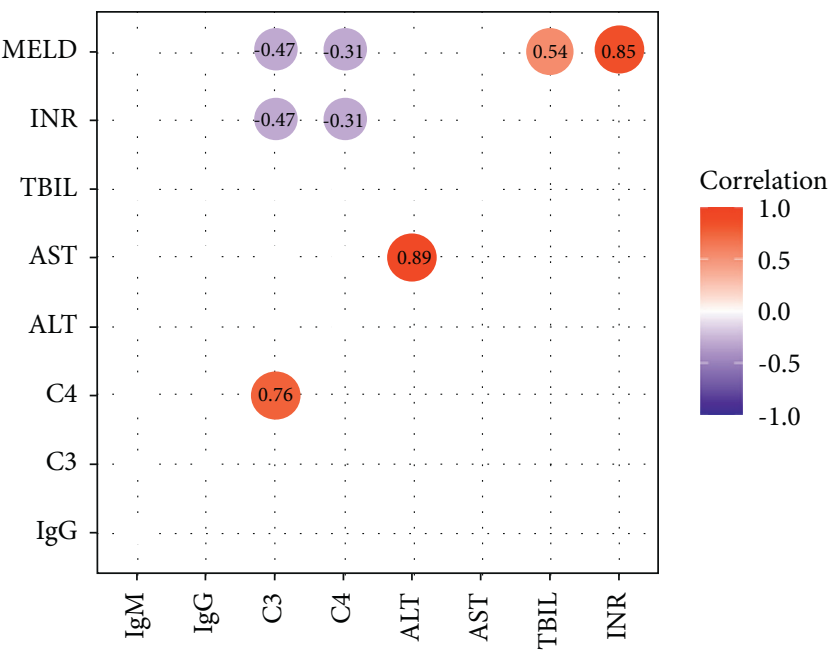

(b)

FIGURE 3: Correlation analysis between immunoglobulin (Ig), complement (C), and model for end-stage liver disease (MELD).

and both $\mathrm{C} 3$ and $\mathrm{C} 4$ were negatively correlated with INR and MELD. In addition, TBIL, INR, WBC, and PLT, but not ANA, resulted as independent risk factors associated with 90-day mortality.

It is speculated that positive autoantibodies in patients with chronic $\mathrm{HBV}$ infection may be associated with a variety of extrahepatic manifestations, while the pathogenesis remains largely unknown [14]. Over-activated CXCR5+CD4+ T cells could help B cells to secret autoantibodies and accelerate liver inflammation [14]. Biopsies in NAFLD revealed that patients with positive autoantibodies were more likely to have mild steatosis than those with negative autoantibodies [6]. In the present study, $\operatorname{IgM}$ was lower in patients with ANA (+), and no significant difference was found in IgG, C3, C4, and extrahepatic manifestation between ANA (+) and ANA (-) patients. INR and MELD scores were significantly lower in ANA (+) groups, indicating that ANA might be a protective factor for ACLF development. Our findings indicated that the MELD score along with WBC and PLT, but not ANA, was independently associated with the overall survival in patients with $\mathrm{HBV}$ ACLF. Overall, it cannot be argued with certainty that positive ANA serves as an additional intrahepatic precipitating event in HBV-related ACLF. Moreover, considering that systemic immunosuppressive therapy is potentially harmful because it may favor the onset of infection [15], 
TABLE 2: Risk factors for 90-day mortality in patients with ACLF.

\begin{tabular}{|c|c|c|c|c|c|c|}
\hline \multirow{2}{*}{ Baseline variables } & \multicolumn{3}{|c|}{ Univariate } & \multicolumn{3}{|c|}{ Multivariate } \\
\hline & Odds ratio & $95 \% \mathrm{CI}$ & $P$ & Odds ratio & $95 \% \mathrm{CI}$ & $P$ \\
\hline Age (years) & 0.988 & $0.949-1.029$ & 0.57 & & & \\
\hline Male & 1.211 & $0.242-6.053$ & 0.82 & & & \\
\hline LC & 1.312 & $0.338-5.094$ & 0.70 & & & \\
\hline $\mathrm{HE}$ & 0.537 & $0.189-1.522$ & 0.24 & & & \\
\hline SBP & 0.592 & $0.150-2.339$ & 0.45 & & & \\
\hline ANA (+) & 0.938 & $0.207-4.259$ & 0.93 & & & \\
\hline ALT & 1.000 & $0.997-1.002$ & 0.66 & & & \\
\hline AST & 1.001 & $0.999-1.004$ & 0.36 & & & \\
\hline TBil & 1.004 & $0.999-1.009$ & 0.09 & 1.005 & $1.000-1.009$ & 0.04 \\
\hline INR & 2.126 & $0.889-5.084$ & 0.09 & 2.559 & $1.215-5.387$ & 0.01 \\
\hline Creatinine & 1.017 & $0.999-1.035$ & 0.06 & & & \\
\hline Serum sodium & 0.891 & $0.780-1.016$ & 0.09 & & & \\
\hline PLT & 0.982 & $0.966-1.000$ & 0.04 & 0.983 & $0.971-0.996$ & $<0.01$ \\
\hline WBC & 1.216 & $0.978-1.510$ & 0.08 & 1.307 & $1.103-1.550$ & $<0.01$ \\
\hline
\end{tabular}

ACLF, acute-on-chronic liver failure; 95\% CI, 95\% confidence interval; LC, liver cirrhosis; ALT, alanine aminotransferase; AST, aspartate aminotransferase; ANA, antinuclear antibodies; CHE, cholinesterase; TBil, total bilirubin; INR, international normalized ratio; PLT, platelet; WBC, white blood cell.

there is no evidence to support empiric steroid-based therapy for ANA (+) HBV-related ACLF.

Data from a large cohort study showed that ANA was positive in $93.4 \%, 49.1 \%, 19.1 \%, 13.9 \%$, and $12.2 \%$ of patients with PBC, AIH, chronic HBV infection, chronic HCV infection, and healthy controls, respectively [16]. For patients with chronic HBV infection and positive ANA, most patients had a titer of $<1: 320$, and the most frequent pattern was homogeneous [16]. A similar result was found in the present study, where ANA titers in HBV-related ACLF were low in most cases. In contrast, the main pattern in ACLF was multiple nuclear dots. The correlation between MRC patterns and liver inflammation remains unclear. It is noteworthy that multiple nuclear dot and rim-like/membranous patterns have a high specificity in patients with $\mathrm{PBC}$, irrespective of the antimitochondrial antibody (AMA) status [17, 18]. AMA was routinely screened at admission, and it was negative in all these patients. Although histopathological evaluation of liver biopsy tissue could not be performed in patients with ACLF due to the coagulation disorders, there is no evidence of $\mathrm{PBC}$ or $\mathrm{AIH}$. Of note, INR is negatively correlated with $\mathrm{C} 3$ and $\mathrm{C} 4$ in patients with ANA (-), while the correlation is not observed in the ANA (+) group; it suggests that the pathogenesis of ACLF may be different between ANA (+) and ANA (-) groups.

The present study has some limitations, such as the lack of histological characteristics and immune cells detection in patients with ANA (+) ACLF. Although IgG, the hallmark of $\mathrm{AIH}$, was not high in ANA (+) patients, it could not exclude patients with co-occurrence of HBV infection and autoimmune liver diseases [19-21]. Considering that the sample size was not large, future large-scale prospective studies are needed to confirm these findings.

\section{Conclusions}

Positive ANA is frequent in HBV-related ACLF, and it does not seem to be associated with poor outcomes, but the pathogenesis of ACLF may be different between ANA (+) and ANA (-) groups.

\section{Abbreviations}

HBV: Hepatitis B virus

ACLF: Acute-on-chronic liver failure

LC: $\quad$ Liver cirrhosis

CHB: $\quad$ Chronic hepatitis B

ANA: Antinuclear antibody

APASL: Asian Pacific Association for the Study of the Liver

NAFLD: Nonalcoholic fatty liver disease

AIH: Autoimmune hepatitis

MRC: Multiple nuclear dots, rim-like, and centromere

HBeAg: Hepatitis B e antigen

MELD: Model for end-stage liver disease

ALT: Alanine aminotransferase

AST: Aspartate aminotransferase

TBil: $\quad$ Total bilirubin

INR: International normalized ratio

WBC: White blood cells

SBP: $\quad$ Spontaneous bacterial peritonitis

HE: Hepatic encephalopathy.

\section{Data Availability}

The data used to support the findings of this study are available from the corresponding author upon request.

\section{Conflicts of Interest}

The authors declare that they have no conflicts of interest.

\section{Authors' Contributions}

Yuan Xue was responsible for study design. Data collection was carried out by Lin Lin, Bin Lin, Qing Lan, Longgen Liu, Jianchun Lu, and Xiujun Zhang. Lin Lin, Bin Lin, and Qing Lan wrote the article, analyzed data, and contributed equally to this study. All authors read and approved the final manuscript. 


\section{Acknowledgments}

This work was supported by the Natural Science Foundation of Jiangsu Province (BK20180183), the Science and Technology Project of Jintan (KJ201925), and Qingmiao Talents Cultivation Project of Changzhou Health Commission (CZQM2020089).

\section{References}

[1] R. Moreau, B. Gao, M. Papp, R. Bañares, and P. S. Kamath, "Acute-on-chronic liver failure: a distinct clinical syndrome," Journal of Hepatology, vol. 75, no. Suppl 1, pp. S27-S35, 2021.

[2] V. Arroyo, R. Moreau, and R. Jalan, "Acute-on-chronic liver failure," New England Journal of Medicine, vol. 382, no. 22, pp. 2137-2145, 2020.

[3] G. Hayat Davoudi, M. Makvandi, A. Teimoori et al., "Frequency of OBI among patients with autoimmune hepatitis," Asian Pacific Journal of Cancer Prevention, vol. 21, no. 9, pp. 2555-2559, 2020.

[4] P. Muratori, A. Granito, M. Lenzi, and L. Muratori, "Limitation of the simplified scoring system for the diagnosis of autoimmune Hepatitis with acute onset," Liver International, vol. 41, no. 3, pp. 529-534, 2021.

[5] P. Loria, A. Lonardo, F. Leonardi et al., "Non-organ-specific autoantibodies in nonalcoholic fatty liver disease: prevalence and correlates," Digestive Diseases and Sciences, vol. 48, no. 11, pp. 2173-2181, 2003.

[6] R. Vuppalanchi, R. J. Gould, R. J. Gould et al., "Clinical significance of serum autoantibodies in patients with NAFLD: results from the nonalcoholic steatohepatitis clinical research network," Hepatology International, vol. 6, no. 1, pp. 379-385, 2012.

[7] A. J. Gilman, A. K. Le, C. Zhao et al., "Autoantibodies in chronic hepatitis $\mathrm{C}$ virus infection: impact on clinical outcomes and extrahepatic manifestations," BMJ Open Gastroenterology, vol. 5, no. 1, Article ID e000203, 2018.

[8] R. Younes, O. Govaere, S. Petta et al., "Presence of serum antinuclear antibodies does not impact long-term outcomes in nonalcoholic fatty liver disease," American Journal of Gastroenterology, vol. 115, no. 8, pp. 1289-1292, 2020.

[9] G. M. Hirschfield, J. K. Dyson, G. J. M. Alexander et al., "The British Society of Gastroenterology/UK-PBC primary biliary cholangitis treatment and management guidelines," Gut, vol. 67, no. 9, pp. 1568-1594, 2018.

[10] A. Granito, P. Muratori, S. Ferri et al., "Diagnosis and therapy of autoimmune hepatitis," Mini Reviews in Medicinal Chemistry, vol. 9, no. 7, pp. 847-860, 2009.

[11] G. Wang, A. Tanaka, H. Zhao et al., "The Asian Pacific association for the study of the liver clinical practice guidance: the diagnosis and management of patients with autoimmune hepatitis," Hepatology International, vol. 15, no. 2, pp. 223-257, 2021.

[12] P. Kamath, R. H. Wiesner, M. Malinchoc et al., "A model to predict survival in patients with end-stage liver disease," Hepatology, vol. 33, no. 2, pp. 464-470, 2001.

[13] S. W. Biggins, W. R. Kim, N. A. Terrault et al., "Evidence-based incorporation of serum sodium concentration into MELD," Gastroenterology, vol. 130, no. 6, pp. 1652-1660, 2006.

[14] Y. Lei, T. Hu, X. Song et al., "Production of autoantibodies in chronic hepatitis $\mathrm{B}$ virus infection is associated with the augmented function of blood CXCR5+CD4+ T cells," PLoS One, vol. 11, no. 9, Article ID e0162241, 2016.
[15] S. Sharma, S. Agarwal, S. Gopi et al., "Determinants of outcomes in autoimmune hepatitis presenting as acute on chronic liver failure without extrahepatic organ dysfunction upon treatment with steroids," Journal of Clinical and Experimental Hepatology, vol. 11, no. 2, pp. 171-180, 2021.

[16] Q. Wei, Y. Jiang, J. Xie et al., "Investigation and analysis of HEp 2 indirect immunofluorescence titers and patterns in various liver diseases," Clinical Rheumatology, vol. 39, no. 8, pp. 2425-2432, 2020.

[17] A. Granito, P. Muratori, C. Quarneti, G. Pappas, R. Cicola, and L. Muratori, "Antinuclear antibodies as ancillary markers in primary biliary cirrhosis," Expert Review of Molecular Diagnostics, vol. 12, no. 1, pp. 65-74, 2012.

[18] A. Granito, P. Muratori, L. Muratori et al., "Antinuclear antibodies giving the 'multiple nuclear dots' or the 'rim-like/ membranous' patterns: diagnostic accuracy for primary biliary cirrhosis," Alimentary Pharmacology and Therapeutics, vol. 24, no. 11-12, pp. 1575-1583, 2006.

[19] A. A. Aljumah, H. Al-Ashgar, H. Fallatah, and A. Albenmousa, "Acute onset autoimmune hepatitis: clinical presentation and treatment outcomes," Annals of Hepatology, vol. 18, no. 3, pp. 439-444, 2019.

[20] M. Lamba, J. H. Ngu, and C. A. M. Stedman, "Trends in incidence of autoimmune liver diseases and increasing incidence of autoimmune hepatitis," Clinical Gastroenterology and Hepatology, vol. 19, no. 3, pp. 573-579, 2021.

[21] A. Islek and H. Keskin, "Seronegative autoimmune hepatitis in children: a single-center experience," Acta Gastro Enterologica Belgica, vol. 84, no. 2, pp. 305-310, 2021. 